\title{
Providing Security against Hole Attack in Manet Victimisation Agglomeration Methodology
}

\author{
P. Anupam, G. Prabhakar
}

\begin{abstract}
Demand of infrastructure less, self-working, self-configuring, communication networks have led to the formation of mobile Adhoc networks (MANET). Manet is extraordinarily valuable over ancient networks in damaging conditions. In Manet all mobile devices work enjoyably for route discover and knowledge transmission. thanks to its broadcast nature of transmission, and cooperative model of operating, routing the traffic may be a tedious task in Manet. Routing protocols square measure perpetually targeted by attackers to wreck network. Routing protocols in Manet ought to be strong against completely different security dangers. Adhoc on-demand distance vector routing (AODV) protocol typically used and studied within the territory of mobile adhoc networks. so as to stop Manet from hole attack a replacement methodology is planned. during this work, hole attack in Manet is detected and prevented by victimisation Hop Count, Reverse Trip Time and Link Length methodology. consistent with the theme, hop count specifies the particular reverse trip time from supply to destination. to seek out the presence of tunnel, the supply can compare calculated reverse trip time with actual reverse trip time and to verify the presence of tunnel, the supply can compare calculated link length with actual link length of the links in ways. This theme provides a security to mobile spontaneous networks from each short still as long hole tunnels. Networksimulator is employed to gauge the performance of mobile spontaneous network. The simulation results show that the planned theme outperformed in terms of output and packet delivery quantitative relation.
\end{abstract}

Keywords:-MANET, AODV, Wormhole Attack, Routing Protocol, Security, Packet Delivery Ratio, Throughput.

\section{INTRODUCTION}

Mobile Ad-hoc Networks (MANET) square measure infrastructure fewer networks therefore security is that the main issue. completely different ways are planned to this point to stop MANETs from numerous types of attacks. Out of those attacks, hole attack is that the main threat. 2 intrusion findion techniques [1] square measure increased that may use clusters and show however clusters are often utilized in order to convey the flexibility to detect hole attack and uninflected them from routing method. at that time 2 routing protocols square measure taken OLSR is Optimized Link State Routing Protocol (proactive) and AODV is Ad-hoc On-Demand Distance Vector Routing Protocol (reactive) so as to seek out that protocol is a lot of at risk of hole attack [2]. The finding shows that AODV is a lot of at risk of hole attack compared to OLSR. Further, a applied mathematics analysis approach is employed and it provides

Revised Manuscript Received on 14 August, 2019.

P.ANUPAM, Assistant Professor, CSE DEPT, Malla Reddy Engineering College for Women, anunithyalohith@gmail.com

G.PRABHAKAR, Assistant Professor, CSE DEPT, Malla Reddy Engineering College for Women, prabhakar.tech@gmail.com higher security and performance as compared to traditional AODV [3], associate degree improvedclustering primarily based approach within which the whole network is divided into completely different clusters and every cluster can have a Cluster Head, that controls all the nodes within the cluster and plays the role of a dominant authority in Manet and Out of band hole attacks that square measure launched by exploiting AODV routing protocol square measure eliminated effectively [4], a light-weight technique is in a position to find and take away the hole attack to a larger extent and provides all-time low total packet loss rate compared with AODV under fire and also the alternative techniques [5], associate degree identity-based signature theme doesn't need distribution of any certificate among nodes therefore it decreases computation overhead and also the performance of the network is evaluated in terms of end-to-end delay, packet delivery quantitative relation, packet loss rate [6]. another techniques [7]-[10] are planned so as to stop hole attacks.

In this paper, hole attack is detected and prevented by victimisation hop count, reverse trip time and link length between the nodes The planned system first of all detects the presence of hole tunnel by victimisation hop count and from the hop count actual reverse trip time is decided that is anon compared by the calculated reverse trip time then detects the hole nodes victimisation link length. The performance of the network is additionally analyzed that shows improved price of output and packet delivery quantitative relation. The network's performance is simulated victimisation NS2 machine.

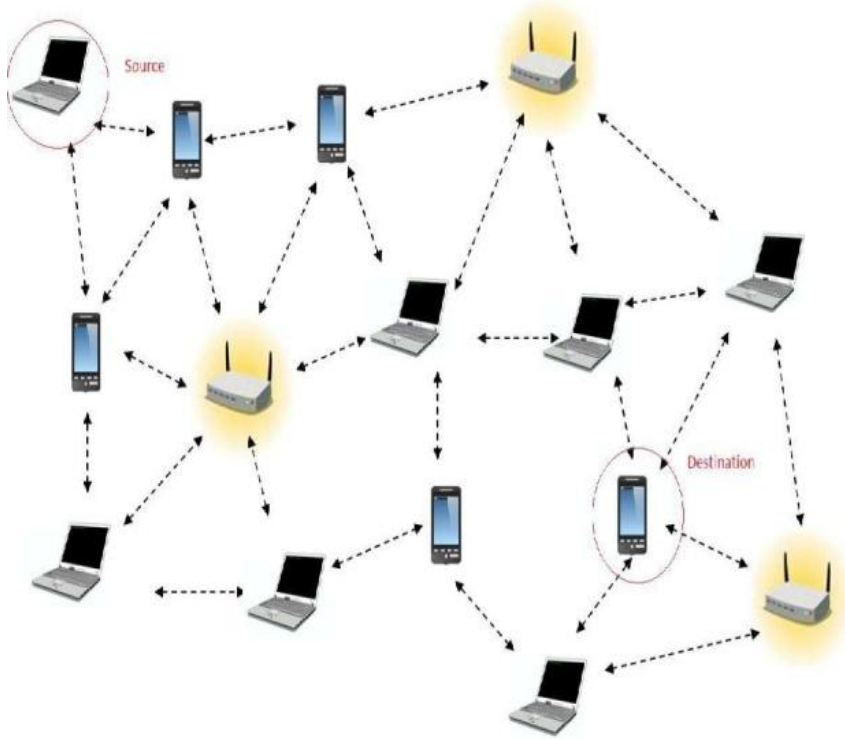

Figure 1. MANET 


\section{BACKGROUND}

Basically, Manet are often classified into initial, second and third generations. the primary generation came up with "packet radio" networks (PRNET), and were sponsored by Defense Advanced Research Projects Agency within the early Seventies. it's evolved to be a sturdy, reliable, operational experimental network. The PRNET used a mixture of acknowledgement and CSMA approaches for medium access, associate degreed a form of distance-vector routing to produce packet-switched networking to mobile field of honor parts in an infrastructure less, hostile atmosphere. The second generation evolved in early 1980's once SURAN (Survivable adaptive Radio Networks) considerably improved upon the radios (making them smaller, cheaper, and power- thrifty), measurability of algorithms, and resilience to electronic attacks. necessary developments throughout this era embrace GloMo (Global Mobile info System) and NTDR ( close to Term Digital Radio) The goal of GloMo was to produce office- atmosphere Ethernet-type transmission property anytime, anywhere, in hand-held devices. Channel access approaches were currently within the CSMA/CA and TDMA molds, and several other novel routing and topology management schemes were developed. The NTDR used agglomeration and link- state routing, and self- organized into a two-tier spontanepous network. currently utilized by the US Army, NTDR is that the solely "real" (non-prototypical) spontanepous network in use these days. The third generation evolved in 1990's additionally termed as industrial network with the arrival of Notebooks computers, open supply software system and equipment's supported RF and infrared. IEEE 802.11 committee adopted the term "ad hoc networks." and also the conception of business (non-military) spontanepous networking had arrived. among the IETF, the Mobile spontanepous Networking (MANET) social unit was horn, and sought-after to standardize routing protocols for spontanepous networks. the event of routing among the Manet social unit and also the larger community forked into reactive (routes on- demand) and proactive (routes ready-to-use) routing protocols 141. The 802.1 1 subcommittee standardized a medium access protocol that was supported collision rejection and tolerated hidden terminals, creating it usable, if not optimum, for building mobile spontanepous network prototypes out of notebooks and802.11 PCMCIA cards. HIPERLAN and Bluetooth were another standards that addressed and benefited spontanepous networking.

\section{AODV PROTOCOL}

The AODV routing protocol is meant for adhoc mobile networks and it will handle uni-cast routing and still as multicast routing $[2,3,4]$. This protocol has the advantageous options of each DSR associate degreed DSDV algorithms and this protocol is an example of On-demand routing protocol which suggests the routes are created only if there's a requirement and additionally it maintains the routes solely as long a they're required. making and maintaining the routes within the network only if they're needed/demand makes this AODV protocol terribly helpful and additionally a decent rule for mobile spontanepous networks (MANET) [5]. All the nodes within the network have routing tables of their own and that they additionally maintain sequence numbers so as to avoid iteration issues [5]. If a supply node needs to send some information to a destination node and if it doesn't have a route to the destination at that point then the supply node broad castes route request (RREQ) packet throughout the network $[2,6]$. The nodes can reply with a RREP if either the destination node or the intermediate node that is on the thanks to notice the destination node. A node that receives the PREQ can send a reply (RREP) providing it's either the destination or if it's a path/route to the destination with a corresponding sequence range and only if that range is bigger than or adequate to the quantity that contains the RREQ [2]. In cases like this the nodes can uni- casts a RREP to the supply, otherwise; the nodes can rerun the RREQ. The nodes can discard the RREQ and don't forward them if they need been processed those already. and also the RREP can discovered forward tips that could the destination by propagating back to the supply nodes $[2,7,8]$ once the supplynode receives the RREP, it records the most recent sequence range to the requested destination and this method is termed as Forward Path setup [9]. The intermediate nodes that receives another RREP once they'd propagated the previous RREP towards the supply, it then checks and compares the new destination sequence range of the new RREP with the previous RREP.

These intermediate nodes updates their routing info and propagates a replacement RREP only if, 1 . The destination sequence range is bigger or two. The new sequence range is same however the hop count is tiny or it'll simply skip the new RREP. This method ensures that this rule isn't creating any loops and solely the foremost effective is chosen [5]. If the info packets keep movement from one node to a different node on a definite path solely then the route remains active otherwise the links can timeout then be deleted from the routing tables of the intermediate nodes. In things like wherever the links break whereas the route is being active then the node upstream of the link break generates a route error (RERR) to the supply node to tell that it's not accessible to the destination (s). once the supply node receives this (RERR) message, then even though the supply node still desires the route then it'll reinitiate the route discovery thereto destination $[4,10]$. Route Discovery Mechanism in AODV: If the supply node "A" needs to initiate communication with destination node "E" as shown within the Figure four.1, then it'll build a association between itself and also the destination and can generate a route request message (RREQ).

This message is then forwarded to the near nodes, and also the near nodes can forward this management message to their near nodes. This method of finding destination node continues till the destination node is found itself or the node that has the recent route to the destination. Once associate degree intermediate node with enough recent routes is found or destination node is found, they generate the route reply message (RREP) and send it back to the supply node. once RREP reaches back to the supply node, a route or the trail is established between the supply node "A" and destination node "E". Once the route is established between " $\mathrm{A}$ " and 
"E", node "A" and "E" will communicate with one another. Figure five depicts the exchange of management messages between supply node and destination node.

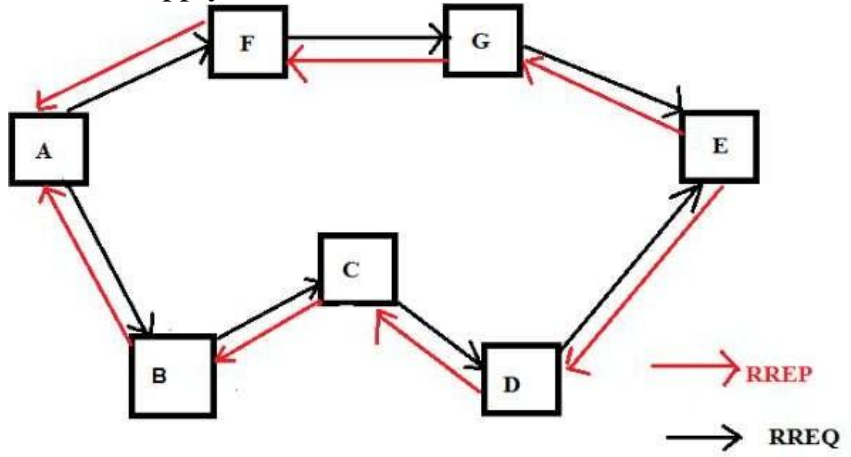

Figure 2. Route Discovery Mechanisms

Route Maintenance Mechanism: once there's a link down or a link breakage between destinations that causes one or over one links unaccessible from the supply node or neighbour's nodes, then the RERR message is generated by the node and sent to the supply node. If there's a route from " $A$ " to " $E$ " via " $D$ ", and if there's a link breakage " $D$ " and "E", then the node "E" can generate and send the RERR message to the supply node " $A$ " informing the supply node that there's a route error. The theme is as shown within the Figure three.

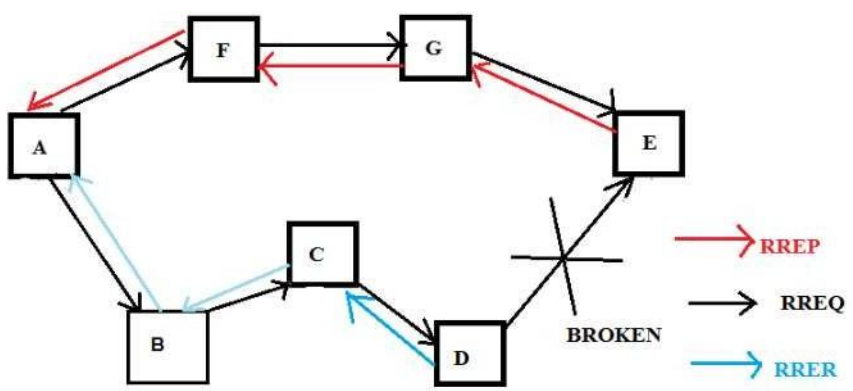

Figure 3. Route Error Message

\section{WORMHOLE ATTACK}

A hole attack is specifically serious attack on Manet routing wherever 2 attackers square measure connected through a high speed off-channel link (tunnel) that square measure critically placed at distinct ends of the network. hole tunnel will then begin gathering the info packets and relay a similar to another location. Malicious nodes can produce associate degree illusion during a network and build real nodes to believe that they're conterminous neighbours. associate degree unwelcome person will collect and manipulate network traffic by attracting and by passing an outsized quantity of network traffic through hole. every ROUTE REQUEST (RREQ) packet is tunnelled to the destination target node of the REQUEST for the appliance of hole attack. traditional routing protocol method is to be followed once the destination node's neighbour hear this REQUEST packet which is able to rerun that duplicate of REQUEST then abandon all alternative received ROUTE REQUEST packets activated from this same Route Discovery. Any routes aside from the routes that square measure being explored are often prohibited by this attack. additionally thereto the attack will even stop routes over 2 hops long from being exposed, if the aggressor is close to the instigator of Route exploration. think

about figure one malicious node that's red in color can broadcast its RREQ to its neighbour nodes that is originally broadcasted to perform a hole attack. Destination node will receive the RREQ request therefore it'll follow a traditional routing method and discard all alternative received ROUTE REQUSET packets. The malicious node can produce a link to a different malicious node that is that the neighbour of the supply node that is dotted here within the figure called hole tunnel. The tunnel successively to be a shortest path to succeed in the destination because it might have less count of hop compared to traditional routes. numerous quite attack like DOS attack, Eavesdropping, and fabrication are often performed with the employment of this privilege. hole attack is in a position to bring down the whole routing system in Manet The aggressor really facilitate helpful services a lot of with efficiency connecting the network, if the aggressor achieves this tunnelling dependably and honestly and no loss is completed. The attack will still be launched even though confidentiality and genuineness is provided over the communication and even though the unwelcome person has no cryptography keys.

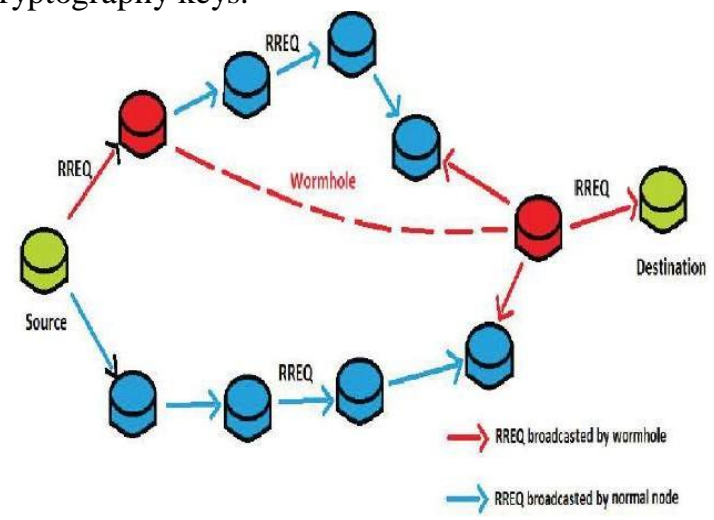

Figure 4. hole state of affairs

\section{RELATED WORK}

There square measure numerous analysis has been planned by researchers in Manet to secure communication network against hole attack. A hole attack is variable in its behaviour associate degreed nature therefore it provides an ample space of exploration and therefore distinct analysis are recommended for the detection of hole attack. a number of review of Literature associated with this work as follows-

Y. C. Hu, A. Perrig, and D. B. Johnson, "Packet leashes: a defense against hole attacks in wireless networks,", 2011.[37]Packet Leash in may be a mechanism to find and defend against hole attacks. The mechanism proposes 2 kinds of leashes for this purpose: Geographic and Temporal. In Geographic Leashes, every node is aware of its precise position and every one nodes have a loosely synchronous clock. Each node, before causation a packet, appends its current position and UTC to that. The receiving node, on receipt of the packet, computes the space to the sender and also the time it took the packet to traverse the trail. The receiver will use this distance anytime info to deduce whether or not the received packet had a hole or not.. once temporal leashes square measure used, the causation node append the time of transmission to every sent packet ts during a packet leash, 
and also the receiving node uses its own packet reception time tr for verification. Thesending node calculates associate degree expiration time te once that a packet shouldn't be accepted, and puts that info within the leash.

T. S a kt hivel a n d R. M. Chandrasekaran. "Detection and interference hole Attack in Manet victimisation Path Tracing Approach".2012[34]The planned work introduced "Detection and interference hole Attack in Manet victimisation Path Tracing Approach". For route discovery, DSR protocol is employed. so as to find the hole, previous per hop distance field, per hop distance field and timestamp fields square measure else to the header of every packet. Author(s) think about each previous per hop distance and per hop distance therefore on compare the distinction between the 2 distances. If the distinction is simply too massive that exceeds the utmost threshold price, then hole is detected. These hole node square measure then isolated from the network.

Priyank Nayak, Akshay Sahay, Yogadhar Pandey "Detection and interference of hole Attacks in MANETs victimisation Detection Packet” 2013 [26] during this work, they gift a general mechanism, while not use of hardware, location info and clock synchronization referred to as detection packet for detective work malicious node in network. Detection Packet has 3 fields: process bit, count to succeed in next hop and time stamp. Timestamp is employed for powerfully detection with correspondence at hole attack. Here detection packet will simply be enclosed within the wide selection of spontanepous routing protocol with solely vital modification within the existing protocol to defend against hole attack. Here DSR protocol is use for route institution and NS-2 for simulations.

RTT - J. Zhen and S. Srinivas. Preventing replay attacks for secure routing in spontanepousnetworks. 2014 [1]during this they delineate regarding specialised hardware together with transmitting aerial for quick causation of 1-bit challenge and distance-bounding protocol securing node trailing are often encountered in multiple hop networks. victimisation special hardware will be a tedious task therefore another approachknown as Time of flight primarily based approach is planned. trip Time [1] mechanism is suggested during this approach. RTT is prolonged as a time that's needed by node A for causation Route Request (RREQ) message to node B receiving time of Route Reply (RREP) message. Node A can determinant among A and every one its near nodes. because the RTT among 2 dishonorable neighbours is bigger than among 2 legitimate neighbours, Node A will simply classify each dishonorable and bonafide neighbours. each node within the network calculates RTT between itself and every one its neighbours. Implementation approach is simple because it doesn't need completely different hardware; in exposed attacks dishonorable neighbours square measure devised therefore, it cannot find exposed attacks.

Jagadhri, Haryana,India et al "Detection of hole attack on AODV Protocol in Mobile Adhoc Networks (MANETS)" 2014 [21] In this paper an answer is planned to stop the network against hole attack. A secret secret's used for cryptography and secret writing of how-do-you-do packets. as a result of this solely authentic nodes can stay within the network, non-authentic nodes (wormhole node) are discarded. As a result communication will turn up solely between the sure nodes. therefore malicious node cannot enter into system and communication is secured. during this work we elect AODV as routing protocol for Manet, a try of hole nodes is chosen for activity hole activity. And simulation is completed on NS two.34 with thirty six nodes. Simulation clearly shows that our methodology is well effective in preventing the network against wormholeattack. DelPHI - D. A. Maltz and D. B. Johnson and Y. Hu. The dynamic supply routing protocol (DSR) for mobile spontanepous, 2015 [2JDelay per Hop(DelPHI)[2] comes as associate degree another approach which may find each exposed and hidden hole attacks additionally called hop count/delay per hop primarily based approach. each attainable disunite route between a supply and a destination square measure determined in urban center. customary delay time perhop on every path is calculated together with its delay time and length of every route hole are often simply discovered with the employment of those values. the trail with a hole tunnel can have higher Delay per Hop (DPH) price. The disadvantage of this methodology is that it doesn't think about quality.

Muhammad Imrana, et al "Analysis of Detection options for hole Attacks in MANETs” 2015 [32/This paper given the options that would be accustomed find the hole attack. These options square measure mentioned well with their execs and cons. The attainable limitations of Intrusion Detection Systems (IDSs) are mentioned. This work provides a basis to create associate degree economical IDS to find hole attacks in MANETs. consistent with our analysis, the techniques supported route request (RREQ) or hop count would be higher than alternative techniques to find hole attacks.

Ashka Shastri, et al, "A hole Attack in Mobile Ad-hoc Network: Detection and Prevention”, 2016. [22] In these planned approach named "Hop primarily based Analysis" the wormholes nodes square measure determined once the explosive decrement within the average hop count of a path from the supply node to destination node had been detected as compared to the opposite ways as a result of the trail with wormholes nodes has smaller hop count.

Mithilesh Kumar et al, "Hop Count primarily based Conjunction management hole Detection Approach for Manet -2016, [30] a straightforward technique for detective work wormholes in spontanepous networks is given within the paper. This methodology employs routing variation between neighbours to see the existence of a hole. The technique is localized, needs solely alittle overhead, and doesn't have special needs like location info, correct synchronization between nodes, special hardware etc. The technique has been tested through simulations ffor completely different|for various\} distributions of nodes for wormholes and different property models. beneath all the evaluated situations, the technique demonstrates wonderful detection possibilitieswith few false alarms that rely upon the worth of threshold. 


\section{PROBLEM STATEMENT}

Mobile Ad-hoc Networks (MANET) square measure infrastructure fewer networks therefore security is that the main issue. completely different ways are planned to this point to stop MANETs from numerous types of attacks. Out of those attacks, hole attack is that the main threat. 2 intrusion findion techniques [1] square measure increased that may use clusters and show however clusters are often utilized in order to convey the flexibility to detect hole attack and uninflected them from routing method. at that time 2 routing protocols square measure taken OLSR is Optimized Link State Routing Protocol (proactive) and AODV is Ad-hoc On-Demand Distance Vector Routing Protocol (reactive) so as to seek out that protocol is a lot of at risk of hole attack [2]. The finding shows that AODV is a lot of at risk of hole attack compared to OLSR. Further, a applied mathematics analysis approach is employed and it provides higher security and performance as compared to traditional AODV [3], associate degree improved agglomeration primarily based approach within which the whole network is divided into completely different clusters and every cluster can have a Cluster Head, that controls all the nodes within the cluster and plays the role of a dominant authority in Manet and Out of band hole attacks that square measure launched by exploiting AODV routing protocol square measure eliminated effectively [4], a light-weight technique is in a position to find and take away the hole attack to a larger extent and provides all-time low total packet loss rate compared with AODV under fire and also the alternative techniques [5], associate degree identity-based signature theme needs distribution of any certificate among nodes therefore it will increase computation overhead and also the performance of the network is analysis are increased in terms of end-to-end delay, packet delivery quantitative relation, packet loss rate [6].There is a requirement to propose some techniques so as to stop hole attacks.

\subsection{Analysis Objective}

The main objective of this work is to attenuate the threat of hole attack in Mobile Ad-hoc Networks by preventing and detective work long still as short hole tunnels within the network.

To reduce the computation overhead arises thanks to analysis of secret key that is distributed among nodes throughout communication.

\section{PROPOSED METHODOLOGY}

The objective of this analysis is to attenuate the threat of hole attack in Mobile Ad-hoc Networks by preventing and detective work long still as short hole tunnels within the network. so as to find and forestall hole attack, H-R-L (Hop Count- Reverse Trip time-Link Length) methodology is employed and also the planned technique works as follows: 1.Source broadcasts Route Request (RREQ) to its neighbour nodes and finds the route to destination.

2.Nodes check their routing table and if no route exists, it rebroadcast RREQ message to its neighbours.

3.The process continues till request reaches to destination and destination can send route reply to supply.
4.Now, supply can check the presence of tunnel within the recommended route \& forwards channel request (CREQ) message to nodes and asks for his or her location coordinates 5.Nodes reply with channel reply (CREP) message and forward their location coordinates to supply.

6.Source calculates Reverse Trip Time (RTT) taken to receive the CREP and if calculated RTT isgreater than actual price, confirms presence of tunnel.

7.Now supply calculates link length of links gift within the path and if calculated link length is over the particular length, hole nodes square measure detected.

8.Source informs all alternative nodes to not communicate with detected hole nodes.

\section{SIMULATION RESULT A.Simulation Setup}

Table 1. The simulation parameters utilized in the work.

\begin{tabular}{|l|l|}
\hline \multicolumn{1}{|c|}{ Parameter } & \multicolumn{1}{c|}{ Value } \\
\hline Channel & Wireless \\
\hline Propagation Model & Two Ray Ground \\
\hline Mobility Model & Random Way Point \\
\hline Routing Protocol & AODV \\
\hline Number of nodes & 50 \\
\hline Mac & 802.11 \\
\hline Antenna & Omni Directional \\
\hline Initial Energy & 50 Joules \\
\hline Network Area & $1300 \mathrm{~m} * 1300 \mathrm{~m}$ \\
\hline Queue Drop & Tail \\
\hline Simulation time & $25 \mathrm{sec}$ \\
\hline Theoretical value & 0.02184 \\
\hline
\end{tabular}

The channel used is wireless and propagation model is 2 ray ground as a result of once the signal received consists of a line of sight and multi-hop elements, it predicts path loss. the quantity of nodes used is fifty and antenna used is position. The queue used is drop tail. during this queue, once the queue is full of most capability then the freshly incoming packets square measure born till queue have ample area to just accept a lot of packets.

\section{B.Packet Delivery quantitative relation}

Figure eight shows the comparison of PDR (packet delivery ratio) of the network achieved once victimisation planned theme and also the existing theme. The planned theme showed the higher price of

packet delivery quantitative relation at zero.95

whereas the worth of packet delivery for the present theme is zero.64. suggests that|this suggests|this implies \} less range of knowledge packets was born once application of planned theme that additionally means the info transmission was a lot of economical and secure. 


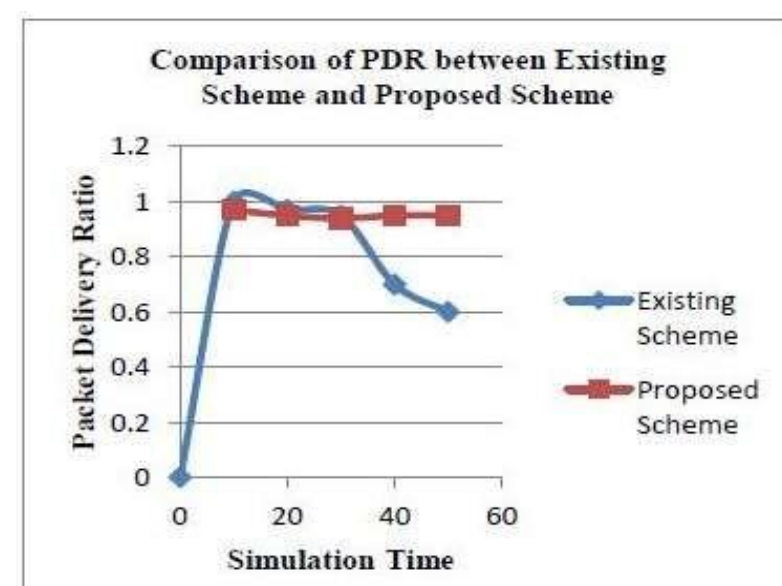

Figure 5. Comparison of Packet Delivery quantitative relation between planned theme and Existing theme

\section{C.Throughput}

Figure six shows the comparison of the output of network achieved once applying the present theme and also the planned theme. The graphs are planned against simulation time that is time taken to simulate the network. the worth of output achieved with our planned theme is thirty six kbps which with the present theme is approx five Kbps. This shows that our planned theme outperforms the present theme.

Comparison of Throughput between

Existing Scheme and Proposed Scheme

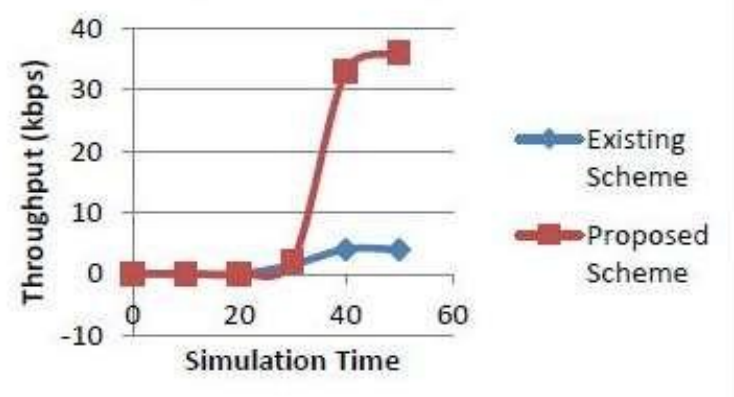

Figure 6. Comparison of output of Network between planned theme and Existing theme

\section{CONCLUSION}

MANET being vulnerable and insecure for various kinds of attacks therefore it needs a decisive, energetic and a secure technique that may be straightaway swollen and use dynamic routing. Security is extremely crucial for Manet. Among all attainable attacks in Manet, hole attack is extremely severe attack because it can considerably degrade network performance to network security. A illegitimate node records and regulate information traffic at one purpose and tunnels it to a plotting node far-flung, which supplies response it regionally that may either brought down the route installation method in hole attack. hole attack may be a terribly dangerous attack several|and lots of|and plenty of researchers have planned many techniques so as to find and forestall MANETs from hole attacks. within the planned work, the technique with success detects and prevents the hole attack for each tunnels short and long tunnels. The performance of the network was analyzed victimisation parameters: packet delivery quantitative relation and output. each these factors tend to point out associate degree improved performance of the network. This shows that the planned theme has performed effectively.

\section{FUTURE SCOPE}

A remarkable chunk of effort has been completed on informative hole attack downside. consistent with the necessity of networks, solutions might vary and selection is out there supported value and wish of security In future, the planned technique are often accustomed find speeding attack within which the nodes rush the route request messages to the destination prior alternative nodes victimisation the tunnel

\section{REFRENCES}

1. Mahdi Nouri, Somayeh Abazari Aghdam, Sajjad Abazari Aghdam, "Collaborative Techniques for detective work hole Attack in MANETs", International Conference on analysis and Innovation in info Systems (ICRIIS), IEEE, Gregorian calendar month 2011.

2. Mohammad Sadeghi, Saadiah Yahya, "Analysis of hole attack on Manets victimisation completely different MANET routing protocols", Fourth International Conference on omnipresent and Future Networks (ICUFN), IEEE, July 2012.

3. Saurabh Upadhyay, Brijesh Kumar Chaurasia, "Detecting and Avoiding hole Attack in Manet victimisation applied mathematics Analysis Approach", Advances in technology and knowledge Technology Networks and Communications, Springer, Vol. 84, pp. 402-408, 2012.

4. J.Anju, C. N. Sminesh, "An Improved Clustering-Based Approach for hole Attack Detection in MANET", three rd International Conference on Ecofriendly Computing and Communication Systems, IEEE, pp. 149-154, 2014.

5. Hosny M. Ibrahim, Nagwa M. Omar, Ebram K. William, “A light-weight Technique to stop hole Attacks in AODV", International Journal of laptop Applications, Vol. 104, Gregorian calendar month 2014.

6. Dhruvi Sharma, Vimal Kumar, Rakesh Kumar, "Prevention of hole Attack victimisation Identity- primarily based Signature theme in M A N E T ", C o m p u t a $\mathrm{t}$ i o $\mathrm{n}$ a 1 Intelligence in data processing, Vol. 2, pp. 475-485, 2015.

7. Juhi Biswas, Ajay Gupta, Dayashankar Singh, "WADP: A hole attack detection and interference technique in Manet victimisation changed AODV routing protocol", ninth International Conference on Industrial and knowledge Systems (ICIIS), IEEE, December 2014.

8. Rajan Patel, Anal Patel, NimishaPatel, "Defending Against hole Attack in MANET", Fifth International Conference on Communication Systems and Network Technologies, IEEE, 2015.

9. .S. B. Geetha, Venkanagouda C Patil, "Evaluating the analysis Trends and Techniques for Addressing hole Attack in MANET", International Journal of laptop Applications, Vol. 110, No. 6, January 2015.

10. Amit Kumar, Sayar Singh Shekhawat, "A Parameter Estimation primarily based Model for Worm Hole Preventive Route Optimization", International Journal of technology and Mobile Computing, Vol. 4, Issue 8, pp. $80-85$, August 2015.

11. RTT - J. Zhen and S. Srinivas. Preventing replay attacks for secure routing in spontanepous networks. In ADHOC-NOW, LNCS 2865

12. DelPHI - D. A. Maltz and D. B. Johnson and Y. Hu. The dynamic supply routing protocol (DSR) for mobile spontanepous.

13. Jyoti Thalor, Ms. Monika, hole Attack Detection and interference Technique in Mobile spontanepous Networks: A Review International Journal of Advanced analysis in technology and software system Engineering, Volume 3, Issue 2, Feb 2013

14. Ajay Prakash Rai, Vineet Srivastava, Rinkoo Bhatia, hole Attack Detection in Mobile spontanepous Networks International Journal of Engineering and Innovative Technology Volume two, Issue 2, August 2012.

15. Reshmi Maulik, Nabendu Chaki, A Study on hole Attacks inMANET International Journal of laptop info Systems and technology Applications, ISSN 2150-7988 Volume three (2011).

16. .Muhammad Imrana, Farrukh Aslam Khanb, Tauseef Jamala, Muhammad Hanif Durad, Analysis of Detection options for hole Attacks in MANETs International Workshop on Cyber Security and Digital Investigation (CSDI 2015).

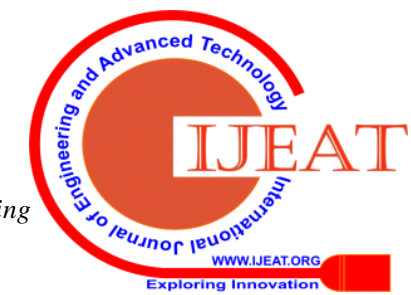


17. Mekhala Chattopadhyay, Mrs. Saswati Mukherjee, associate degree Approach To find hole Attack In Aodv primarily based Manet Jadavpur University Kolkata-70003.

18. Detection and interference of hole attacks in MANETs victimisation path tracing approach,http://www.academia.edu/ six seven seven one eight seven six / Detection_and_Prevention_of_Worm hole_Attacks_in_MANETs_using_Pa th_Tracing_Approach.

19. Marianne Azer, Sherif El-Kassas, Magdy El-Soudani, A Full Image of the hole Attacks Towards Introducing complicated hole Attacks in wireless spontanepous Networks International Journal of technology and knowledge Security, Vol. 1, No. 1, May 2009.

20. Manjeet Singh, Gaganpreet Kaur, "A Surveys of Attacks in MANET" International Journal of Advanced analysis in technology and software system Engineering Volume three, Issue 6, June 2013.

21. Jagadhri, Haryana,India et al "Detection of hole attack on AODV Protocol in Mobile Adhoc Networks (MANETS)" International Journal Of Engineering And technology ISSN:2319-7242Volume three Issue nine Sept 2014 Page No. 7979-7985. 